\title{
Ex vivo susceptibility and genotyping of Plasmodium falciparum isolates from Pikine, Senegal
}

\author{
Aminata Mbaye ${ }^{1^{*}} \mathbb{D}$, Amy Gaye ${ }^{1}$, Baba Dieye ${ }^{1}$, Yaye D. Ndiaye ${ }^{1}$, Amy K. Bei $^{1,2}$, Muna Affara $^{4}$, Awa B. Deme ${ }^{1}$, \\ Mamadou S. Yade', Khadim Diongue', Ibrahima M. Ndiaye', Tolla Ndiaye' ', Mouhamed Sy', Ngayo Sy', \\ Ousmane Koita ${ }^{5}$, Donald J. Krogstad ${ }^{3}$, Sarah Volkman², Davis Nwakanma ${ }^{4}$ and Daouda Ndiaye ${ }^{1,2}$
}

\begin{abstract}
Background: The monitoring of Plasmodium falciparum sensitivity to anti-malarial drugs is a necessity for effective case management of malaria. This species is characterized by a strong resistance to anti-malarial drugs. In Senegal, the first cases of chloroquine resistance were reported in the Dakar region in 1988 with nearly $7 \%$ population prevalence, reaching $47 \%$ by 1990 . It is in this context that sulfadoxine-pyrimethamine temporarily replaced chloroquine as first line treatment in 2003, pending the introduction of artemisinin-based combination therapy in 2006. The purpose of this study is to assess the ex vivo sensitivity to different anti-malarial drugs of the P. falciparum population from Pikine.

Methods: Fifty-four samples were collected from patients with non-complicated malaria and aged between 2 and 20 years in the Deggo health centre in Pikine in 2014. An assay in which parasites are stained with 4', 6-di-amidino2-phenylindole (DAPI), was used to study the ex vivo sensitivity of isolates to chloroquine, amodiaquine, piperaquine, pyrimethamine, and dihydroartemisinin. High resolution melting was used for genotyping of pfdhps, pfdhfr, pfmdr 1, and pfcrt genes.

Results: The mean $\mathrm{IC}_{50} \mathrm{~s}$ of chloroquine, amodiaquine, piperaquine, dihydroartemisinin, and pyrimethamine were, respectively, 39.44, 54.02, 15.28, 2.23, and $64.70 \mathrm{nM}$. Resistance mutations in pfdhfr gene, in codon 437 of pfdhps gene, and an absence of mutation at position 540 of pfdhps were observed. Mutations in codons K76T of pfcrt and N86Y of pfmdr 1 were observed at 51 and $11 \%$ population prevalence, respectively. A relationship was found between the K76T and N86Y mutations and ex vivo resistance to chloroquine.
\end{abstract}

Conclusion: An increase in sensitivity of isolates to chloroquine was observed. A high sensitivity to dihydroartemisinin was observed; whereas, a decrease in sensitivity to pyrimethamine was observed in the parasite population from Pikine.

Keywords: Chemosensitivity, Genotyping, Plasmodium falciparum, Pikine

\section{Background}

Malaria is a parasitic disease which was responsible for nearly 429,000 deaths worldwide in 2015. More than $92 \%$ of these deaths occur in Africa in children [1]. Children under 5 years and pregnant women are among those

\footnotetext{
*Correspondence: aminatambaye155@gmail.com

1 Laboratory of Parasitology/Mycology HALD, Cheikh Anta Diop University of Dakar, PO Box 5005, Dakar, Senegal

Full list of author information is available at the end of the article
}

most vulnerable to malaria. This can be explained by the fact that in malaria-endemic areas, after years of exposure, non-sterilizing immunity develops that is protective against severe malaria. Pregnant women tend to lose this acquired immunity due to the immune-suppression which occurs during pregnancy, and studies have shown that placental parasitaemia in these individuals is higher than those of peripheral blood [2], putting both mother and child at high risk. Many tools are currently available to fight malaria in these vulnerable groups such as 
intermittent preventive treatment of pregnant women with sulfadoxine-pyrimethamine (SP) $[3,4]$ and seasonal malaria chemoprevention (SMC) for children under 5 years of age in areas with seasonal transmission of malaria [5]. Currently in Senegal, for SMC in children, the drug regimen of choice is SP-amodiaquine. Amodiaquine is also used in combination with artesunate for treatment of uncomplicated malaria. Cross-resistance has been observed between amodiaquine and chloroquine. Thus, it would be important to monitor the sensitivity of parasites to SP to ensure the effectiveness of these preventive combination treatments. A good correlation between in vivo resistance to $\mathrm{SP}$ and in vitro resistance has been characterized as well as a strong association between in vivo resistance and single nucleotide polymorphisms in pfdhps (Plasmodium falciparum dihydropteroate synthetase) and pfdhfr (P. falciparum dihydrofolate reductase) genes [6-10]. In vitro resistance to pyrimethamine is associated with the mutation at codon S108N of $p f d h f r$ gene, whereas the resistance to sulfadoxine is associated with the mutation K540E of the pfdhps gene. In West Africa, triple mutations at codons N51I, C59R and S108N of the pfdhfr gene and the mutation $\mathrm{G} 437 \mathrm{~A} / \mathrm{T}$ in the $p f d h p s$ gene are frequently observed. In Senegal, the quadruple mutation representing the triple mutation of the pfdhfr gene plus the mutation G437A/T in the pfdhps is also frequently observed [11-13]. In East Africa, a further mutation in pfdhps at codon K540E has been described [9]. This quintuple mutation is highly associated with a therapeutic failure to SP. WHO recommends that in areas where the quintuple mutation reaches greater than $50 \%$ population prevalence that SP use should be abandoned for chemoprevention of malaria [2].

The monitoring of amodiaquine, chloroquine, piperaquine, and dihydroartemisinin sensitivity is a clear priority in the fight against malaria. As amodiaquine is used for prevention and treatment of malaria in Senegal, it is important to determine if there is a decrease of sensitivity of $P$. falciparum population to this drug and if this decrease is related to the past (cross-resistance between amodiaquine and chloroquine) or current amodiaquine use. The overall objective of this study is to assess the ex vivo sensitivity of $P$. falciparum isolates from Pikine to SP, amodiaquine, chloroquine, piperaquine, and dihydroartemisinin.

\section{Methods}

\section{Sample collection}

In 54 children aged between 5 and 20 years of age who came for consultation at the Deggo health centre in Pikine in 2014, both venous blood and filter paper were collected. These children suffered from non-complicated malaria with confirmation by drop thick and thin smears. Informed consent by the child and or guardian was requested before any samples were taken. The study protocol was validated by the Human Subjects Committee of Tulane University and the Ethics Committee of the Ministry of Health of Senegal. The work is funded by the International Centres of Excellence for Malaria Research, (ICEMR) West Africa (U19AI089696).

\section{Ex vivo assays \\ Drug preparation}

Pyrimethamine (Sigma), chloroquine diphosphate salt, amodiaquine hydrochloride, dihydroartemisinin, and piperaquine were reconstituted with dimethyl sulfoxide (DMSO). The dilution was performed with the nonsupplemented Roswell Park Memorial Institute Medium (RPMI). Twofold serial dilutions were performed with the non-supplemented Roswell Park Memorial Institute Medium (RPMI). The highest drug concentrations plated were $750 \mathrm{nM}$ for chloroquine, $500 \mathrm{nM}$ for piperaquine, $100 \mathrm{nM}$ for amodiaquine, $50 \mathrm{nM}$ for dihydroartemisinin, and 295,056 nM for pyrimethamine. Each drug concentration was plated in duplicate. Plates were frozen at $-20^{\circ} \mathrm{C}$ until required.

\section{Culture and $\mathrm{Cl} 50$ determination}

The tubes of venous blood collected for DAPI test were transported to Aristide Le Dantec Hospital within $6 \mathrm{~h}$ of blood draw. The plasma was removed by centrifugation ( $2500 \mathrm{~g}$ for $10 \mathrm{~min})$. The pellet was then washed twice with unsupplemented RPMI by centrifugation at $2500 \mathrm{~g}$ for $5 \mathrm{~min}$. Parasitaemia was adjusted to between 0.4 and $1 \%$ and haematocrit was adjusted to $2 \%$. The parasitaemia and haematocrit adjusted parasite mixture was distributed on the previously dosed 96-well drug plates and incubated in the presence of gas $\left(94 \% \mathrm{~N}_{2}, 5 \% \mathrm{CO}_{2}, 1 \%\right.$ $\mathrm{O}_{2}$ ) [14] at $37{ }^{\circ} \mathrm{C}$. After $48 \mathrm{~h}$ of incubation, the growth of parasite in positive control wells specifically plated for microscopic evaluation was checked. The assays was determined to be complete when parasites had reinvaded as new rings. Plates were frozen at $-20{ }^{\circ} \mathrm{C}$ until reading and reading was performed for all plates at once.

For staining and reading the DAPI assay, $100 \mu \mathrm{l}$ of membrane lysis buffer containing the molecule DAPI was distributed to each well. After a $30 \mathrm{~min}$ incubation, plates were centrifuged $4000 \mathrm{~g}$ for $10 \mathrm{~min}$, washed with PBS, and fluorescence was measured using a Fluoroskant Ascent. $\mathrm{IC}_{50}$ values were calculated using graph Pad Prism software version 5 . Reference clone $3 \mathrm{D} 7$, sensitive to all antimalarial drugs tested, was used for each batch of drug plates as a positive control. 

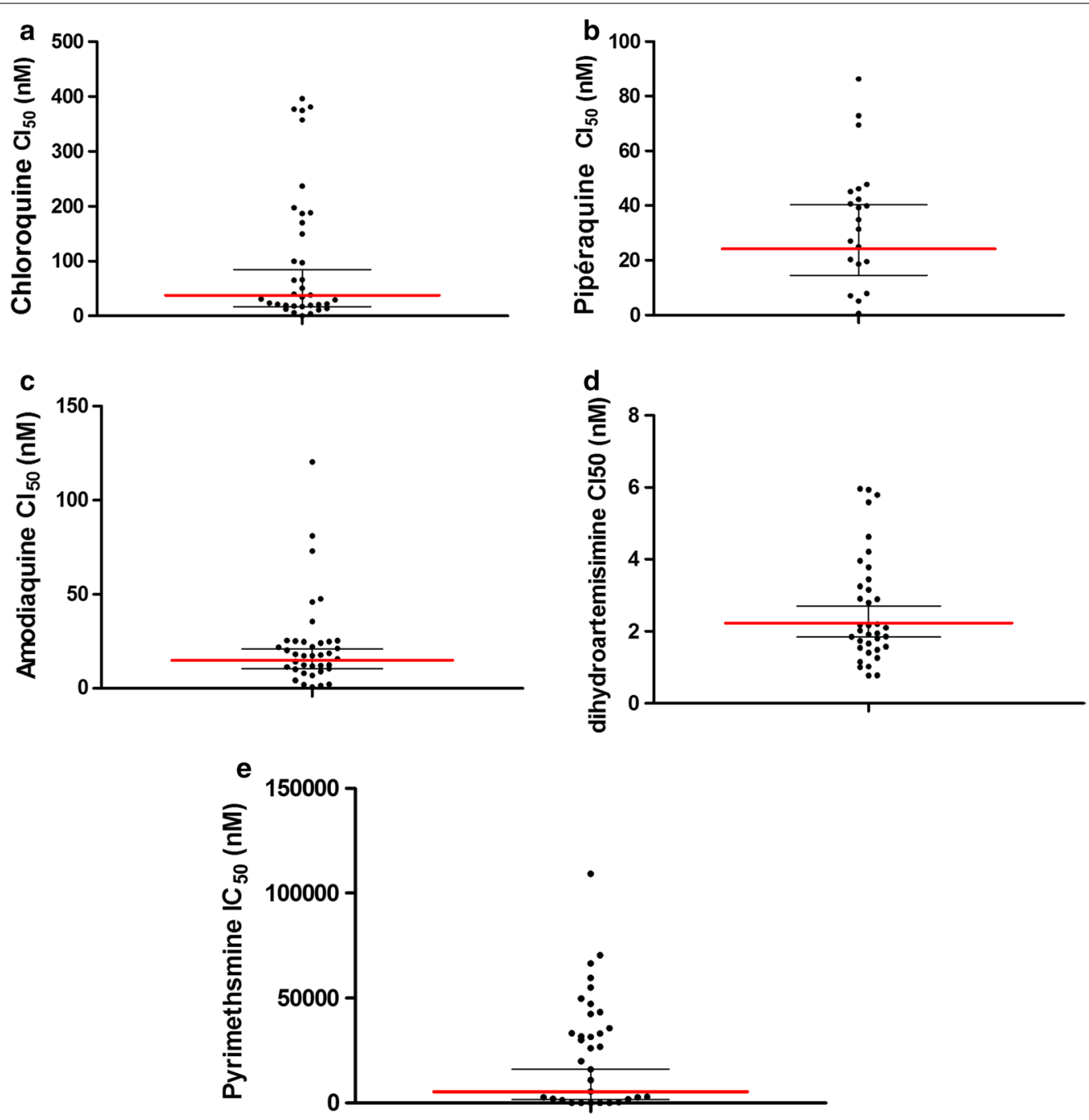

Fig. 1 Distribution of $\mathrm{IC}_{50}$ value among parasites collected in Pikine in 2014 and tested again Chloroquine (a), Piperaquine (b), Amodiaquine (c), Dihydroartemisinin $(\mathbf{d})$ and Pyrimethamine $(\mathbf{e})$. Horizontal lines indicate the geometric mean of $I C_{50}$ value in red with the $95 \%$ Cl. For all panels $I C_{50}$ high value is not represented

DNA extraction and single nucleotide polymorphism typing Parasitic DNA was extracted using the QIAamp DNA Blood Mini kit (Qiagen) according to manufacturer instructions. Codons 51, 59-108 of the pfdhfr gene, 436, 437, 540, and 613 of pfdhps, 76 of pfcrt and 86 of pfmdr1 were genotyped by HRM [12]. Glass capillaries were used with a $10 \mu \mathrm{l}$ final volume. All PCR were performed using 2.5X LightScanner master mix (Biofire), with forward primers at a final concentration of $0.05 \mu \mathrm{M}$, reverse primers at a final concentration of $0.2 \mu \mathrm{M}$ (asymmetric PCR), allele specific probes at a final concentration of $0.2 \mu \mathrm{M}$, and $1 \mu \mathrm{l}$ of genomic DNA, as previously described [12].
Standard software included with the instruments was used for unlabelled probe analysis to visualize melting peaks based on different melting temperatures, indicative of different base pairs, and compared with controls to call alleles for a given assay.

\section{Statistical analysis}

The Graph-pad Prism Software version 5 was used to calculate the $\mathrm{IC}_{50}$ value for all drugs for each parasite isolate tested. For each codon position, the distribution of $\mathrm{IC}_{50} \mathrm{~s}$ were compared using the Mann-Whitney $\mathrm{U}$ test. The test is significant if the P value is less than 0.05 . 


\section{Results}

Ex vivo sensitivity to chloroquine, amodiaquine, piperaquine, dihydroartemisinin, and pyrimethamine Good sensitivity of the 3D7 reference strain to chloroquine $(22.05 \mathrm{nM})$ was observed. The geometric mean $\mathrm{IC}_{50}$ for all isolates for chloroquine, amodiaquine, piperaquine, dihydroartemisinin, and pyrimethamine were $35.44,54.02,15.28,2.23$, and $64.70 \mathrm{nM}$, respectively (Fig. 1).

\section{Prevalence of point mutations of pfdhps, pfdhfr, pfcrt, and $p f m d r 1$ genes}

Mutations at codons 51, 59 and 108 of dhfr were highly prevalent with 100, 95 and $96 \%$ observed, respectively. For codon 437, a proportion of $44 \%$ with mixed wild type and mutant $(2 \%)$ was observed. No mutation was observed at the 540 position of pfdhps gene. For codon 76 of the pfcrt gene $51 \%$ of mutations and $6 \%$ of mixed was found. About the codon 86 of $p f m d r 1$, the mutation rate amounted to $11 \%$ (Table 1).

All strains that contained the mutation N86Y of $p f m d r 1$ gene were mutant or mixed at codon K76T of pfcrt. For pfdhps and pfdhfr triple, quadruple and quintuple mutation were observed at 95,39 and $0 \%$ prevalence, respectively (Table 2).

Table 1 Prevalence of point mutation of pfdhps, pfdhfr, pfcrt, and pfmdr1 genes at positions 51, 59, 108, 437, 540, 76 , and 86

\begin{tabular}{llll}
\hline Gene & Codon & Allele & Prevalence \\
\hline Pfdhfr & N51I & I & $100 \%(51 / 51)$ \\
& C59R & R & $96 \%(49 / 51)$ \\
& S108N & N & $98 \%(50 / 51)$ \\
& & Mixte & $2 \%(1 / 51)$ \\
Pfdhps & A437G & G & $44 \%(24 / 54)$ \\
& & G/A & $2(1 / 54)$ \\
& K540E & K & $100 \%(54 / 54)$ \\
Pfcrt & K76T & T & $0 \%$ \\
& & Mixte & $51 \%(28 / 540)$ \\
Pfmdr1 & N86Y & Y & $11 \%$ \\
& & Mixte & $0 \%$ \\
\hline
\end{tabular}

Table 2 Prevalence of mutant haplotype in pfdhps and pfdhfr

\begin{tabular}{lc}
\hline Haplotype & Prevalence \\
\hline Triple mutation & $95 \%(40 / 42)$ \\
Quadruple mutation & $39 \%(15 / 38)$ \\
Quintuple mutation & $0 \%(0 / 44)$ \\
\hline
\end{tabular}

Table 3 Correlation between mutation at Pfcrt codon K76T and Pfmdr1 codon N86Y and sensitivity to chloroquine, amodiaquine, piperaquine, dihydroartemisinin, and pyrimethamine

\begin{tabular}{lllll}
\hline Compound & Codon & $\begin{array}{l}\text { GM of IC50 } \\
\text { (nM) for WA }\end{array}$ & $\begin{array}{l}\text { GM of IC50 } \\
\text { (nM) for MA }\end{array}$ & P value \\
\hline Chloroquine & K76T & 13.86 & 74.15 & 0.0195 \\
& N86Y & 33.90 & 391.2 & 0.0279 \\
Amodiaquine & K76T & 9.924 & 10.47 & 0.0539 \\
& N86Y & 9.417 & 20.69 & 0.5290 \\
Piperaquine & K76T & 56.16 & 46.45 & 0.9370 \\
& N86Y & 27.15 & 58.00 & 0.8155 \\
Dihydroartemisinin & K76T & 2.139 & 1.773 & 0.4614 \\
& N86Y & 2.165 & 1.906 & 0.7727 \\
Pyrimethamine & K76T & 11,596 & 4981 & 0.8366 \\
& N86Y & 2.165 & 3.377 & 0.4605 \\
\hline
\end{tabular}

$P$ value is significant when less than 0.05

$G M$ : geometric mean, $I_{50}$ : half maximal inhibitory concentration, WA: wild allele, MA: mutant allele

\section{Association between genotype and phenotype}

The ex vivo resistance to chloroquine has been found to be linked to the mutation at codon K76T of pfcrt gene and N86Y of pfmdr1 (Table 3). For amodiaquine, the observation was that the values found for the mutant strains on these codons were higher but not significantly different. In our study, no relationship was found between the mutation $\mathrm{K} 76 \mathrm{~T}$ and N86Y and the decrease in ex vivo sensitivity to piperaquine, dihydroartemisinin and pyrimethamine. High prevalent of mutation on codon 51, 59 and 108 of the $p f d h f r$ gene was found, probably related to the decrease in sensitivity to pyrimethamine.

\section{Discussion}

It is essential to have effective anti-malarial drugs to fight malaria. Artemisinin combination therapies were introduced as first-line therapy in this context. However, growing resistance to ACT has been observed in Southeast Asia: in Cambodia in 2006, Myanmar and Thailand in 2008, and Vietnam in 2009, and Laos in 2013 [15]. The rationale for monitoring resistance phenotypically by the in vitro method is that several anti-malarial drugs can be tested at the same time, and the evolution of the sensitivity or resistance of parasite populations to drugs either in use or no longer in use can be studied. The study of molecular markers of resistance informs the level of resistance of the Plasmodium population to drugs at the genetic level. This will result in better understanding of which drugs to monitor in vivo, which combinations to avoid, and those that can be used effectively for the management of malaria. In Senegal, pyrimethamine combined with sulfadoxine is used for intermittent preventive treatment for pregnant women. Further SP, plus 
amodiaquine is used for preventive seasonal treatment for children under 5 years old in areas with high transmission of malaria [16]. Chloroquine was eliminated in Senegal in 2003 following cases of resistance in vivo [17]. Piperaquine combined with dihydroartemisinin is used for the third-line treatment of non-complicated malaria. For molecular markers of resistance, the mutation on codons K76T of pfcrt gene and N86Y of pfmdr1 has been demonstrated to be associated with resistance to chloroquine [18-22]. Resistance to amodiaquine is associated with the N86Y mutation and cases of crossresistance between amodiaquine and chloroquine have been observed. For pyrimethamine, the mutation on codon $\mathrm{S} 108 \mathrm{~N}$ is strongly associated with resistance [23]. In vivo resistance of $P$. falciparum to chloroquine has been confirmed in Pikine, Moulomp (Casamance) and Fatick [24]. Indeed, the emergence of resistance to chloroquine in Senegal were reported in 1988 in Dakar with 5.7\% therapeutic failure [25]. These cases then increased to $47.5 \%$ in 1990 and $25-30 \%$ in 1992 in Pikine [26], leading to the withdrawal of chloroquine for treatment of non-complicated malaria in Senegal in 2003. However, amodiaquine, which has some cross-resistance with chloroquine, is always used in combination for the treatment or prevention of malaria. In Dakar in 2010 a geometric mean of $41.63 \mathrm{nM}$ for chloroquine and $19.4 \mathrm{nM}$ for amodiaquine was found with another ex vivo technique [13]. At Pikine, an in vitro sensitivity study conducted in 2000 showed $31 \%$ of resistance to chloroquine with a geometric mean of $272 \mathrm{nM}$ [27]. In 2001, a geometric mean of $135 \mathrm{nM}$ was registered [28]. Prevalence of mutation of $51 \%$ on codon K76T of pfcrt and $11 \%$ on the N86Y of pfmdr1 gene was recorded in 2014.

The prevalence of the $76 \mathrm{~T}$ allele in isolates from Pikine was $72.4 \%$ when chloroquine was used (2000-2003), $47.16 \%$ during the period of the use of amodiaquine-SP for first-line treatment (2004-2005) and 59.46\% with ACT used between 2006 and 2009. N86Y mutation had decreased between 2005 and 2009 and it was about 20\% in 2009 [29]. A selection of N86 and K76 alleles were noted in Thiès, another region in Senegal, in 2013 [30]. The results of this study have shown that the mutation on codon N86Y was related to the decrease in sensitivity to chloroquine. For amodiaquine, the geometric mean of the isolates with the mutation N86Y was higher compared to isolates with wild-type allele, but the difference was not significant. For piperaquine, no relationship between genotype and $\mathrm{IC}_{50}$ was observed. An association was found between the presence of the $76 \mathrm{~T}$ allele and the decrease in sensitivity to chloroquine ( $\mathrm{p}=0.0195)$ but not to amodiaquine (0.0539) and piperaquine $(0.9370)$. A decrease in ex vivo sensitivity and an increase in the prevalence of the N86Y mutation relationship was not significantly found with amodiaquine ( $\mathrm{p}=0.5290)$ and the geometric mean for piperaquine was very low compared to that found in other countries [30-34]. These compounds are currently used in combination with dihydroartemisinin for piperaquine, SP and artesunate for amodiaquine. Good ex vivo sensitivity of isolates to dihydroartemisinin was found, implying continued effectiveness of one of the partner drugs of ACT used in the treatment of non-complicated malaria in Pikine.

Used since 2003 in Senegal, first as temporary replacement of chloroquine for the treatment of non-complicated malaria, SP is now used for preventive treatment of malaria. The results showed low ex vivo sensitivity of isolates to pyrimethamine. This was accompanied by a high prevalence of mutations in codons N51I, C59R and $\mathrm{S} 108 \mathrm{~N}$ of the pfdhfr gene. A strong presence of mutation on codon S108N (67 and 24\%) and 51/59 (40 and $20 \%)$ were recorded, respectively, for Thiès in 2003 and Pikine in 2002. At Pikine, 65\% (N51I), 61\% (C59R) and $78 \%(\mathrm{~S} 108 \mathrm{~N})$ of mutation was found [35]. These results suggest that resistance to pyrimethamine emerged before the introduction of the SP association. The double mutation 437/540 of the pfdhps gene has been demonstrated as being related to resistance to sulfadoxine [10]. Ex vivo sensitivity to sulfadoxine of isolates has not been studied. However, an absence of mutation on codon K540E and high prevalence of mutation at codon G437A/T has been recorded, which confirms the efficacy of sulfadoxine. On the other hand, studies revealed that triple mutation $108-59-51$ is strongly associated with resistance to SP in African isolates [36] and that the presence of the double mutation $437 / 540$ indicates a high risk of treatment failure in SP [9]. An increase of triple mutation and quadruple mutation in Pikine was noted [35], but the quintuple mutation was absent. However, mutation of $2.12 \%$ at codon 540 in pfdhfr was found in Dakar [36].

\section{Conclusion}

Monitoring the sensitivity of $P$. falciparum populations to anti-malarial drugs is a necessity for effective malaria case management. An increase in the sensitivity of isolates to chloroquine Good efficacy of dihydroartemisinin amodiaquine and piperaquine and decrease in sensitivity to pyrimethamine were observed.

\footnotetext{
Abbreviations

SP: sulfadoxine-pyrimethamine; ACT: artemisinin-based combination therapy; Pfdhps: Plasmodium falciparum dihydropteroate synthetase; Pfdhfr: Plasmodium falciparum dihydrofolate reductase; WHO: World Health Organization; ICEMR: International Centres of Excellence for Malaria Research; DMSO: dimethyl sulfoxide; RPMI medium: Roswell Park Memorial Institute medium; HRM: high resolution melting; DNA: deoxy ribo nucleic acid; PCR: polymerase chain
} 
reaction; Pfcrt: Plasmodium falciparum chloroquine resistance transporter; Pfmdr1: Plasmodium falciparum multidrug resistance protein 1; CQ: chloroquine; AMQ: amodiaquine; PQ: piperaquine; DHA: dihydroartemisinin.

\section{Authors' contributions}

$A M, B D, A G, Y D N, A B D, M S Y, I M N$, TN, AKB carried out the experiments and collected data. DN, OK, DK, SV, DN conceived and designed the study. AM and $A K B$ analysed the data. AM, BD and KD wrote the manuscript. All authors read and approved the final manuscript.

\section{Author details \\ ${ }^{1}$ Laboratory of Parasitology/Mycology HALD, Cheikh Anta Diop Univer- sity of Dakar, PO Box 5005, Dakar, Senegal. ${ }^{2}$ Department of Immunology and Infectious Diseases, Harvard School of Public Health, Boston, MA, USA. ${ }^{3}$ Tulane University, New Orleans, LA, USA. ${ }^{4}$ Medical Research Council Unit, The Gambia, Fajara, Gambia. ${ }^{5}$ University of Bamako, Bamako, Mali.}

\section{Acknowledgements}

We would like to acknowledge the International Centre for Excellence in Malaria Research (ICEMR) project and the Parasitology and Mycology Laboratory Le Dantec Hospital. We thank Cyrille Diedhiou, Nasserdine Papa Nze, Dior Diop, Younouss Diedhiou, Lamine Ndiaye, Amadou Mactar Mbaye, the Degoo patients and staff for their contribution to this study.

\section{Competing interests}

The authors declare that they have no competing interests.

\section{Consent for publication}

The participants in this study consented publication.

\section{Ethics approval and consent to participate}

The Human Subjects Committee of Tulane University and the Ethics Committee of the Senegal Ministry of Health in Dakar both approved the protocols used in these studies.

\section{Funding}

The work was supported by the International Centers of Excellence for Malaria Research, (ICEMR) West Africa (U19AI089696).

\section{Publisher's Note}

Springer Nature remains neutral with regard to jurisdictional claims in published maps and institutional affiliations.

Received: 23 January 2017 Accepted: 6 June 2017 Published online: 14 June 2017

\section{References}

1. WHO. World Malaria Report. Geneva: World Health Organization; 2016

2. Bricaire F, Danis M, Gentilini M. Paludisme et grossesse. Cahiers. Santé. 2008;63:289-92.

3. Bamba S, Séré A, Nikiéma R, Halidou T, Thiéba B, Dao B, et al. Traitement préventif intermittent à la sulfadoxine-pyriméthamine du paludisme chez les femmes enceintes: efficacité et observance dans deux hôpitaux urbains du Burkina Faso. Pan Afr Med J. 2013;14:105.

4. Rupérez M, González R, Mombo-Ngoma G, Kabanywanyi AM, Sevene E, Ouédraogo S, et al. Mortality, morbidity, and developmental outcomes in infants born to women who received either mefloquine or sulfadoxine-pyrimethamine as intermittent preventive treatment of malaria in pregnancy: a cohort study. PLoS Med. 2016;13:e1001964.

5. OMS. Chimioprévention Du Paludisme Saisonnier. 2013. p. 1-45. Available from: http://www.who.int/malaria/areas/preventive_therapies/children/ $\mathrm{fr} /$. Accessed 9 June 2017.

6. Happi CT, Gbotosho GO, Folarin OA, Akinboye DO, Yusuf BO, Ebong OO, et al. Polymorphisms in Plasmodium falciparum dhfr and dhps genes and age related in vivo sulfadoxine-pyrimethamine resistance in malariainfected patients from Nigeria. Acta Trop. 2005:95:183-93.
7. Hailemeskel E, Kassa M, Taddesse G, Mohammed H, Woyessa A, Tasew $\mathrm{G}$, et al. Prevalence of sulfadoxine-pyrimethamine resistance-associated mutations in dhfr and dhps genes of Plasmodium falciparum three years after SP withdrawal in Bahir Dar, Northwest Ethiopia. Acta Trop. 2013;128:636-41.

8. Nzila AM, Mberu EK, Sulo J, Dayo H, Winstanley PA, Sibley CH, et al. Towards an understanding of the mechanism of pyrimethamine-sulfadoxine resistance in Plasmodium falciparum: genotyping of dihydrofolate reductase and dihydropteroate synthase of Kenyan parasites. Antimicrob Agents Chemother. 2000;44:991-6.

9. Naidoo I, Roper C. Mapping, "partially resistant", "fully resistant", and "super resistant" malaria. Trends Parasitol. 2013;29:505-15.

10. Abdul-Ghani R, Farag HF, Allam AF. Sulfadoxine-pyrimethamine resistance in Plasmodium falciparum: a zoomed image at the molecular level within a geographic context. Acta Trop. 2013;125:163-90.

11. Ndiaye D, Dieye B, Ndiaye YD, Van Tyne D, Daniels R, Bei AK, et al. Polymorphism in dhfr/dhps genes, parasite density and ex vivo response to pyrimethamine in Plasmodium falciparum malaria parasites in Thies, Senegal. Int J Parasitol Drugs Drug Resist. 2013;3:135-42.

12. Daniels R, Ndiaye D, Wall M, McKinney J, Séne PD, Sabeti PC, et al. Rapid, field-deployable method for genotyping and discovery of single-nucleotide polymorphisms associated with drug resistance in Plasmodium falciparum. Antimicrob Agents Chemother. 2012;56:2976-86.

13. Fall B, Pascual A, Sarr FD, Wurtz N, Richard V, Baret E, et al. Plasmodium falciparum susceptibility to anti-malarial drugs in Dakar, Senegal, in 2010: an ex vivo and drug resistance molecular markers study. Malar J. 2013;12:107.

14. Bei AK, Patel SD, Volkman SK, Ahouidi AD, Ndiaye D, Mboup S, et al. An adjustable gas-mixing device to increase feasibility of in vitro culture of Plasmodium falciparum parasites in the field. PLoS ONE. 2014;9:e90928.

15. WHO. Status report on artemisinin resistance [Internet]. 2014. p. 1-8. Available from: http://www.who.int/malaria/publications/atoz/statusrep-artemisinin-resistance-sep2014.pdf. Accessed 9 June 2017.

16. PNLP. Plan Strategique National 2011-2015. 2010. p. 129. Available from: http://www.pnlp.sn/wp-content/uploads/2016/08/PNLP_PSN_VFF_0302-2016.pdf. Accessed 9 June 2017.

17. Mouzin E, Thior PM, Diouf MB, Samd B. Focus sur le Senegal. 2010. Available from: http://www.rollbackmalaria.org/files/files/resources/report4-fr. pdf. Accessed 9 June 2017.

18. Payne D. Spread of chloroquine resistance in Plasmodium falciparum. Parasit Today. 1987;3:241-6.

19. Lim P, Chy S, Ariey F, Incardona S, Chim P, Sem R, et al. pfcrt Polymorphism and chloroquine resistance in Plasmodium falciparum strains isolated in Cambodia. Antimicrob Agents Chemother. 2003:47:87-94.

20. Sidhu AB, Verdier-Pinard D, Fidock DA. Chloroquine resistance in Plasmodium falciparum malaria parasites conferred by pfcrt mutations. Science. 2002;298:210-3.

21. Eyase $F L$, Akala HM, Ingasia $L$, Cheruiyot A, Omondi A, Okudo C, et al. The role of pfmdr1 and pfcrt in changing chloroquine, amodiaquine, mefloquine and lumefantrine susceptibility in Western-Kenya P. falciparum samples during 2008-2011. PLoS ONE. 2013;8:e64299.

22. Pradines B, Dormoi J, Briolant S, Bogreau H, Rogier C. La résistance aux antipaludiques. Revue Francophone des Laboratoires. 2010;422:51-62.

23. Basco LK, Ringwald P. Molecular epidemiology of malaria in Yaounde, Cameroon. Vl. Sequence variations in the Plasmodium falciparum dihydrofolate reductase-thymidylate synthase gene and in vitro resistance to pyrimethamine and cycloguanil. Am J Trop Med Hyg. 2000;62:271-6.

24. Gaye O, Bah IB, Diallo S, Victorius A, Bengua E, Faye OFO. The emergence of chloroquine-resistant malaria in Dakar. Senegal. Ann Soc Belg Med Trop. 1990;70:33-7.

25. Trape JF, Legros F, Ndiaye P, Konate L, Bah IB, Diallo S, et al. Chloroquineresistant Plasmodium falciparum malaria in Senegal. Trans $\mathrm{R}$ Soc Trop Med Hyg. 1989;83:761-3.

26. Sokhna CS, Molez JF, Ndiaye P, Sane BTJ. In vivo chemosensitivity tests of Plasmodium falciparum to chloroquine in Senegal: the development of resistance and the assessment of therapeutic efficacy. Bull Soc Pathol Exot. 1997;90:83-9.

27. Penh P, Thomas SM, Ndir O, Dieng T, Mboup S, Wypij D, et al. In vitro chloroquine susceptibility and PCR analysis of pfcrt and pfmdr1 polymorphisms in Plasmodium falciparum isolates from Senegal. Am J Trop Med Hyg. 2002;66:474-80 
28. Dieng T, Bah IB, Ndiaye PM, Diallo I, Diop BM, Brasseur P, et al. Evaluation de la sensibilité in vitro de Plasmodium falciparum à la chloroquine par le deli-microtest dans la région de Dakar (Sénégal). Med Trop (Mars). 2005:65:580-3.

29. Ly O, Gueye PEO, Deme AB, Dieng T, Badiane AS, Ahouidi AD, et al. Evolution of the pfcrt T76 and pfmdr1 Y 86 markers and chloroquine susceptibility 8 years after cessation of chloroquine use in Pikine, Senegal. Parasitol Res. 2012;11:1541-6.

30. Mbaye A, Dieye B, Ndiaye YD, Bei AK, Muna A, Deme AB, et al. Selection of N86F184D1246 haplotype of Pfmrd1 gene by artemether-lumefantrine drug pressure on Plasmodium falciparum populations in Senegal. Malar J. 2016;15:433.

31. Basco LK, Ringwald P. In vitro activities of piperaquine and other 4-aminoquinolines against clinical isolates of Plasmodium falciparum in Cameroon. Antimicrob Agents Chemother. 2003;47:1391-4.

32. Nsobya SL, Kiggundu M, Nanyunja S, Joloba M, Greenhouse B, Rosenthal PJ. In vitro sensitivities of Plasmodium falciparum to different antimalarial drugs in Uganda. Antimicrob Agents Chemother. 2010;54:1200-6.
33. Barends M, Jaidee A, Khaohirun N, Singhasivanon P, Nosten F. In vitro activity of ferroquine (SSR 97193) against Plasmodium falciparum isolates from the Thai-Burmese border. Malar J. 2007;6:81.

34. Mwai L, Kiara SM, Abdirahman A, Pole L, Rippert A, Diriye A, et al. In vitro activities of piperaquine, lumefantrine, and dihydroartemisinin in Kenyan Plasmodium falciparum isolates and polymorphisms in pfcrt and pfmdr1. Antimicrob Agents Chemother. 2009;53:5069-73.

35. Ndiaye D, Daily JP, Sarr O, Ndir O, Gaye O, Mboup S, et al. Mutations in Plasmodium falciparum dihydrofolate reductase and dihydropteroate synthase genes in Senegal. Trop Med Int Health. 2005;4:1176-9.

36. Boussaroque A, Fall B, Madamet M, Wade KA, Fall M, Nakoulima A, et al. Prevalence of anti-malarial resistance genes in Dakar, Senegal from 2013 to 2014. Malar J. 2016;15:347.

\section{Submit your next manuscript to BioMed Central and we will help you at every step:}

- We accept pre-submission inquiries

- Our selector tool helps you to find the most relevant journal

- We provide round the clock customer support

- Convenient online submission

- Thorough peer review

- Inclusion in PubMed and all major indexing services

- Maximum visibility for your research

Submit your manuscript at www.biomedcentral com/submit 